\title{
REGULAR AUSLANDER-REITEN COMPONENTS CONTAINING DIRECTING MODULES
}

\author{
ANDRZEJ SKOWROŃSKI
}

(Communicated by Maurice Auslander)

\begin{abstract}
We describe completely all regular Auslander-Reiten components of artin algebras containing directing modules. We prove also that the AuslanderReiten quiver of an artin algebra admits at most finitely many DTr-orbits containing directing modules.
\end{abstract}

\section{INTRODUCTION}

Throughout the paper $A$ will denote a fixed artin algebra over a commutative artin ring $R$ and $n$ will be the rank of the Grothendieck group $K_{0}(A)$ of $A$. By a module we always mean a finitely generated right module. We shall denote by $\bmod A$ the category of all (finitely generated right) $A$-modules, by $\operatorname{rad}(\bmod A)$ the $\operatorname{radical}$ of $\bmod A$, and by $\operatorname{rad}^{\infty}(\bmod A)$ the intersection of all powers $\operatorname{rad}^{i}(\bmod A), i \geq 0, \operatorname{of} \operatorname{rad}(\bmod A)$. From the existence of the Auslander-Reiten sequences in $\bmod A$ we know that $\operatorname{rad}(\bmod A)$ is generated by the irreducible maps as a left and as a right ideal (see [1]). We denote by $\mathrm{D}$ the standard duality $\operatorname{Hom}_{R}(-, I)$, where $I$ is the injective envelope of $R / \operatorname{rad} R$ in $\bmod R$. Further, we denote by $\Gamma_{A}$ the Auslander-Reiten quiver of $A$ and by $\tau_{A}, \tau_{A}^{-}$the Auslander-Reiten operators $\mathrm{DTr}, \operatorname{TrD}$ on $\bmod A$, respectively. We will not distinguish between an indecomposable $A$-module, its isomorphism class, and the vertex in $\Gamma_{A}$ corresponding to it. A connected component $\mathscr{C}$ of $\Gamma_{A}$ is said to be regular if $\mathscr{C}$ contains neither a projective nor an injective module. Following [13], a connected component $\mathscr{C}$ of $\Gamma_{A}$ is said to be generalized standard if $\operatorname{rad}^{\infty}(X, Y)=0$ for all modules $X$ and $Y$ from $\mathscr{C}$. Finally, following [9], an indecomposable $A$-module $M$ is called directing if it does not belong to a cycle $M \rightarrow M_{1} \rightarrow \cdots \rightarrow M_{r} \rightarrow M$ of nonzero nonisomorphisms between indecomposable $A$-modules.

Directing modules have played an important role in the representation theory of artin algebras: preprojective and preinjective components in general and

Received by the editors January 2, 1992 and, in revised form, April 8, 1992; the contents of this paper were presented at the CMS Annual Seminar/NATO Advanced Research Workshop on "Representations of Algebras and Related Topics" (Carleton University, Ottowa, August, 1992) and at the RIMS Symposium "Representation Theory of Finite Groups and Finite Dimensional Algebras" (Kyoto, October, 1991).

1991 Mathematics Subject Classification. Primary 16G70; Secondary 16G10.

Research partially supported by Polish Scientific Grant KBN No. 1222/2/91. 
connecting components of tilted algebras consist entirely of directing modules. It was proved in [11] that a hereditary algebra $H$ of type $\Delta$ has a regular tilting module if and only if the quiver $\Delta$ has at least three vertices and is neither of Euclidean nor of Dynkin type. Moreover, if $T$ is a regular tilting $H$-module and $B=\operatorname{End}_{H}(T)$ is the associated tilted algebra, then the connecting component of $\Gamma_{B}$ is a regular component consisting entirely of directing modules and is of the form $Z \Delta^{\mathrm{op}}$, where $\Delta^{\mathrm{op}}$ is the opposite quiver of $\Delta$. In [14] the author and Smalø proved that such components exhaust all regular components consisting entirely of directing modules.

We shall prove here the following theorem on the structure of regular Auslander-Reiten components of artin algebras containing directing modules.

Theorem. Let $\mathscr{C}$ be a regular connected component of $\Gamma_{A}$ containing a directing module. Write $A=P \oplus Q$, where the simple direct summands of $P / \operatorname{rad} P$ are exactly the simple composition factors of modules in $\mathscr{C}$. Denote by $t_{Q}(A)$ the ideal of $A$ generated by all images of all maps from $Q$ to $A$, and put $B=A / t_{Q}(A)$. Then

(i) $\mathscr{C}$ has only finitely many $\tau_{A^{-}}$orbits and all modules from $\mathscr{C}$ are directing.

(ii) $\mathscr{C}$ is a generalized standard component of $\Gamma_{A}$.

(iii) $t_{Q}(A)$ is the annihilator of $\mathscr{C}$ in $A$.

(iv) $B$ is a tilted algebra of the form $\operatorname{End}_{H}(T)$ with $H$ a (wild) hereditary artin algebra and $T$ a regular tilting $H$-module.

(v) $\mathscr{C}$ is the connecting component of $\Gamma_{B}$.

In the course of the proof, we obtain a condition for an indecomposable $A$-module $M$, in terms of its neighbourhood in $\Gamma_{A}$, to be nondirecting. In particular, we obtain the following consequence of this criterion and $[4,15]$.

Corollary 1. Let $\mathscr{C}$ be a regular connected component of $\Gamma_{A}$ having infinitely many $\tau_{A}$-orbits. Then for any module $M$ in $\mathscr{C}$ there exists a cycle $M \rightarrow M_{1} \rightarrow$ $\cdots \rightarrow M_{k} \rightarrow M$ in $\bmod A$ with $M_{1}, \ldots, M_{k}$ from $\mathscr{C}$.

As another consequence of this criterion and the above theorem we obtain the following result.

Corollary 2. $\Gamma_{A}$ admits at most finitely many $\tau_{A}$-orbits containing directing modules.

Combining this corollary with a result in [5] we also obtain the following fact.

Corollary 3. Let $A$ be representation-infinite and $R$ be an algebraically closed field. Then $\Gamma_{A}$ admits infinitely many $\tau_{A}$-orbits without directing modules.

The results of this paper have been partially announced in the author's survey article [12].

\section{Preparatory lemmas}

We shall use the following well-known fact from [1].

Lemma 1. Let $I$ be an ideal in $A, B=A / I$, and $M$ be a $B$-module. Then $\tau_{B} M$ is a submodule of $\tau_{A} M$.

The following lemma will play a crucial role in our investigations. 
Lemma 2. Let $M_{1}, \ldots, M_{r}$ be pairwise nonisomorphic indecomposable $A$ modules such that $\operatorname{Hom}_{A}\left(M_{i}, \tau_{A} M_{j}\right)=0$ for all $1 \leq i, j \leq r$. Let $M=$ $M_{1} \oplus \cdots \oplus M_{r}, I$ be the annihilator of $M$ in $A$, and $B=A / I$. Then $M$ is $a$ partial tilting $B$-module. In particular, $r \leq n$.

Proof. We have from our assumption that $\operatorname{Hom}_{A}\left(M, \tau_{A} M\right)=0$, and then, by Lemma $1, \operatorname{Hom}_{B}\left(M, \tau_{B} M\right)=0$. Hence $\operatorname{Ext}_{B}^{1}(M, M) \cong \bar{D}_{\overline{H o m}}\left(M, \tau_{B} M\right)=$ 0 . Clearly, $M$ is a faithful $B$-module. This implies that $p d_{B} M \leq 1$ (see $[8,(1.5)])$. Indeed, it is enough to show that $\operatorname{Hom}_{B}\left(\mathrm{D} B, \tau_{B} M\right)=0$ (see [9, (2.4)]). Since $M$ is a faithful $B$-module, DM is a faithful $B^{\text {op }}$-module, and we have a monomorphism $B^{\text {op }} \rightarrow(\mathrm{D} M)^{s}$ for some $s \geq 1$. Then there is an epimorphism $M^{s} \rightarrow \mathrm{D} B$ in $\bmod B$, and consequently $\operatorname{Hom}_{B}\left(\mathrm{D} B, \tau_{B} M\right)=0$ because $\operatorname{Hom}_{B}\left(M, \tau_{B} M\right)=0$. Therefore, $M$ is a partial tilting $B$-module and, according to a result of Bongartz [3], $M$ may be extended to a tilting $B$-module. Hence $r$ is less than or equal to the rank of $K_{0}(B)$, which is again less than or equal to $n$. This finishes the proof.

By a path in $\bmod A$ we mean a sequence $X_{0} \rightarrow X_{1} \rightarrow \cdots \rightarrow X_{s-1} \rightarrow X_{s}$ of nonzero nonisomorphisms between indecomposable $A$-modules. A path $M_{0} \rightarrow$ $M_{1} \rightarrow \cdots \rightarrow M_{r-1} \rightarrow M_{r}$ in $\Gamma_{A}$ is called sectional if $M_{i-2} \neq \tau_{A} M_{i}$ for each $i$, $2 \leq i \leq r$.

The following lemma gives some sufficient conditions for an indecomposable $A$-module $M$ to be nondirecting.

Lemma 3. Let $M$ be an indecomposable A-module and $\mathscr{C}$ the connected component of $\Gamma_{A}$ containing $M$. Assume that there is a sectional path $M=M_{0} \rightarrow$ $M_{1} \rightarrow \cdots \rightarrow M_{n-1} \rightarrow M_{n}$ in $\mathscr{C}$ such that the following conditions are satisfied:

(i) The modules $M_{1}, \ldots, M_{n}$ are nonprojective and pairwise nonisomorphic.

(ii) For any $i, 1 \leq i \leq n-1$, and any sectional path in $\mathscr{C}$ of the form $U_{t} \rightarrow U_{t-1} \rightarrow \cdots \rightarrow U_{1}=\tau_{A} M_{i+1} \rightarrow U_{0}=M_{i}$ such that $t \leq n$, the modules $U_{1}, \ldots, U_{t}$ are nonprojective and nonisomorphic to the modules $M_{0}, M_{1}, \ldots, M_{n}$.

Then there is a cycle $M \rightarrow X_{1} \rightarrow \cdots \rightarrow X_{k} \rightarrow M$ in $\bmod A$ with $X_{1}, \ldots, X_{k}$ from $\mathscr{C}$.

Proof. Obviously we may assume that $M_{0} \not M_{i}$ for each $i, 1 \leq i \leq n$. Then, by (i), the modules $M_{0}, \ldots, M_{n}$ are pairwise nonisomorphic, and, by Lemma 2 , there are $0 \leq p, q \leq n$ such that $\operatorname{Hom}_{A}\left(M_{p}, \tau_{A} M_{q}\right) \neq 0$. In particular, we have in $\bmod A$ a path $M_{p} \rightarrow \tau_{A} M_{q} \rightarrow M_{q-1}$, if $q \geq 1$, and a path $M_{p} \rightarrow \tau_{A} M_{0} \rightarrow \tau_{A} M_{1} \rightarrow M_{0}$, if $q=0$. Let $r$ be the least number with $1 \leq r \leq n$ and such that there is in $\bmod A$ a path

$$
M_{i}=Z_{s} \stackrel{f_{s}}{\rightarrow} Z_{s-1} \rightarrow \cdots \rightarrow Z_{2} \stackrel{f_{2}}{\rightarrow} Z_{1}=\tau_{A} M_{r} \stackrel{f_{1}}{\rightarrow} Z_{0}=M_{r-1}
$$

with $Z_{1}, \ldots, Z_{s}$ from $\mathscr{C}$ and $0 \leq i \leq n$. We shall show that $r=1$. In this case, we have in $\bmod A$ a required cycle $M=M_{0} \rightarrow \cdots \rightarrow M_{i}=Z_{s} \rightarrow$ $\cdots \rightarrow Z_{1} \rightarrow Z_{0}=M$. Suppose that $r \geq 2$. We claim that there is a path (*) with $s>n, f_{1}, \ldots, f_{n}$ irreducible, $Z_{1}, \ldots, Z_{s}$ from $\mathscr{C}$, and $Z_{j} \neq$ $\tau_{A} Z_{j-2}$ for each $j, 2 \leq j \leq n$. We may assume that, if $f_{i}$ does not belong to $\operatorname{rad}^{\infty}(\bmod A)$, then $f_{i}$ is irreducible, because otherwise we replace it by a finite sequence of irreducible maps. Suppose now that some $f_{i}$ belongs to 
$\operatorname{rad}^{\infty}(\bmod A)$ and let $m$ be the least index with this property. Then there is in $\bmod A$ an infinite sequence of irreducible maps

$$
\cdots V_{j+1} \stackrel{g_{j+1}}{\rightarrow} V_{j} \stackrel{g_{j}}{\rightarrow} \cdots \rightarrow V_{1} \stackrel{g_{1}}{\rightarrow} V_{0}=Z_{m-1}
$$

such that all $V_{j}$ are indecomposaible and $\operatorname{Hom}_{A}\left(Z_{m}, V_{j}\right) \neq 0$ for all $j \geq 0$. But then we have in $\bmod A$ a path

$$
\begin{aligned}
M_{i} & =Z_{s} \stackrel{f_{s}}{\rightarrow} \cdots \rightarrow Z_{m} \stackrel{g_{n}}{\rightarrow} V_{n-1} \rightarrow \cdots \rightarrow V_{1} \stackrel{g_{1}}{\rightarrow} V_{0} \\
& =Z_{m-1} \stackrel{f_{m-1}}{\rightarrow} \cdots \rightarrow Z_{1} \stackrel{f_{1}}{\rightarrow} Z_{0},
\end{aligned}
$$

where $f_{1}, \ldots, f_{m-1}, g_{1}, \ldots, g_{n}$ are irreducible, and all modules forming this path belong to $\mathscr{C}$. Finally, suppose that there is $a$ such that $a<n, a<s$, $f_{1}, \ldots, f_{a}$ are irreducible, $Z_{j} \neq \tau_{A} Z_{j-2}$ for each $2 \leq j \leq a$, and $Z_{a+1} \cong$ $\tau_{A} Z_{a-1}$. Then from (i) and (ii), the modules $Z_{0}, \ldots, Z_{a-1}$ are nonprojective, and consequently we have in $\bmod A$ a path

$$
M_{i}=Z_{s} \rightarrow \cdots \rightarrow Z_{a+1} \cong \tau_{A} Z_{a-1} \rightarrow \cdots \rightarrow \tau_{A} Z_{0} \rightarrow M_{r-2},
$$

a contradiction to our choice of $r$. Similarly, if $(*)$ is a sectional path in $\Gamma_{A}$, then $s>n$ because $Z_{s}=M_{i}$ and from our assumption (ii). Therefore, we proved the existence of a path $(*)$ with $s>n, f_{1}, \ldots, f_{n}$ irreducible, $Z_{j} \not \tau_{A} Z_{j-2}$ for each $2 \leq j \leq n$, and $Z_{1}, \ldots, Z_{s}$ from $\mathscr{C}$. Then, by (i) and (ii), the modules $Z_{0}, Z_{1}, \ldots, Z_{n}$ are nonprojective. We claim that they are also pairwise nonisomorphic. Indeed, if $Z_{k} \cong Z_{h}$ for some $n \geq k>h \geq 0$, then, by a result of Bautista and Smalø [2], we have $Z_{h+1} \cong \tau_{A} Z_{k-1}$, and hence again a path in $\bmod A$ of the form

$$
M_{i}=Z_{s} \rightarrow \cdots \rightarrow Z_{h+1} \cong \tau_{A} Z_{k-1} \rightarrow \cdots \rightarrow \tau_{A} Z_{0} \rightarrow M_{r-2},
$$

a contradiction. Finally, by Lemma 2, there are then $b$ and $c, 0 \leq b, c \leq n$, such that $\operatorname{Hom}_{A}\left(Z_{b}, \tau_{A} Z_{c}\right) \neq 0$, and we have in $\bmod A$ a path of the form

$$
M_{i}=Z_{s} \rightarrow \cdots \rightarrow Z_{b} \rightarrow \tau_{A} Z_{c} \rightarrow \cdots \rightarrow \tau_{A} Z_{0} \rightarrow M_{r-2},
$$

which again contradicts our choice of $r$. Therefore, $r=1$. This finishes our proof.

For a connected component $\mathscr{C}$ of $\Gamma_{A}$, we denote by $\mathscr{C}_{S}$ the stable part of $\mathscr{C}$, obtained from $\mathscr{C}$ by removing the $\tau_{A}$-orbits of projective and injective modules.

The following lemma shows possible applications of Lemma 3.

Lemma 4. Let $\mathscr{C}$ be a connected component of $\Gamma_{A}$ and $\mathscr{D}$ be a connected component of $\mathscr{C}_{S}$. Assume that $\mathscr{D}$ has infinitely many $\tau_{A}$-orbits and no oriented cycles. Let $M$ be a module in $\mathscr{D}$ such that the length of any walk in $\mathscr{C}$ from a nonstable module to $M$ is at least $2 n$. Then there is a sectional path $M=M_{0} \rightarrow M_{1} \rightarrow \cdots \rightarrow M_{n}$ in $\mathscr{C}$ such that $\mathscr{C}$ has no paths of the form $M_{i}=Z_{s} \rightarrow Z_{s-1} \rightarrow \cdots \rightarrow Z_{1}=\tau_{A} M_{j}$ with $s \leq n$ and $0 \leq i, j \leq n$. In particular, there is a cycle $M \rightarrow X_{1} \rightarrow \cdots \rightarrow X_{k} \rightarrow M$ in $\bmod A$ with $X_{1}, \ldots, X_{k}$ from $\mathscr{C}$.

Proof. Since $\mathscr{D}$ is a stable translation quiver with infinitely many $\tau_{A}$-orbits and without oriented cycles, we infer from [4, 15] (see also [6]), that $\mathscr{D} \cong Z \Delta$ for some infinite, locally finite, connected, valued quiver $\Delta$ without oriented cycles. 
Let $\mathscr{O}$ be a fixed $\tau_{A}$-orbit in $\mathscr{D}$. We claim that $\mathscr{D}$ contains a path from $M$ to a module in $\mathscr{O}$. Indeed, since $\mathscr{D}$ is connected, there is a walk $M=Y_{0}-Y_{1}-$ $\cdots-Y_{r}$ in $\mathscr{D}$ with $Y_{r}$ from $\mathscr{O}$, where $Y_{i}-Y_{i+1}$ means either $Y_{i} \rightarrow Y_{i+1}$ or $Y_{i} \leftarrow Y_{i+1}$. We may assume $r \geq 1$. Suppose that there is a path in $\mathscr{D}$ from $M$ to $\tau_{A}^{-q} Y_{i}$ for some $q \geq 0,0 \leq i \leq r-1$. Then either $\tau_{A}^{-q} Y_{i} \rightarrow \tau_{A}^{-q} Y_{i+1}$ or $\tau_{A}^{-q} Y_{i} \rightarrow \tau_{A}^{-q-1} Y_{i+1}$ is an arrow in $\mathscr{D}$, and consequently $\mathscr{D}$ contains either a path from $M$ to $\tau_{A}^{-q} Y_{i+1}$ or a path from $M$ to $\tau_{A}^{-q-1} Y_{i+1}$. Then the claim follows by induction on $i, 0 \leq i \leq r$.

Consider now the set $\mathscr{P}$ of all paths $M=N_{0} \rightarrow N_{1} \rightarrow \cdots \rightarrow N_{k}$ of finite length in $\mathscr{D}$ starting at $M$ and such that $\tau_{A} N_{k}$ is not a successor of $M$ in $\mathscr{D}$. Observe that, if $M=N_{0} \rightarrow N_{1} \rightarrow \cdots \rightarrow N_{k}$ is a path from $\mathscr{P}$, then $\mathscr{D}$ has no paths from $N_{i}$ to $\tau_{A} N_{j}, 0 \leq i, j \leq k$. Indeed, if $N_{i}=V_{0} \rightarrow V_{1} \rightarrow$ $\cdots \rightarrow V_{p}=\tau_{A} N_{j}$ is a path in $\mathscr{D}$, then $\mathscr{D}$ admits a path

$$
\begin{aligned}
M & =N_{0} \rightarrow N_{1} \rightarrow \cdots \rightarrow N_{i}=V_{0} \rightarrow V_{1} \rightarrow \cdots \rightarrow V_{p} \\
& =\tau_{A} N_{j} \rightarrow \tau_{A} N_{j+1} \rightarrow \cdots \rightarrow \tau_{A} N_{k},
\end{aligned}
$$

a contradiction since $\tau_{A} N_{k}$ is not a successor of $M$ in $\mathscr{D}$. In particular, the paths $M=N_{0} \rightarrow N_{1} \rightarrow \cdots \rightarrow N_{m}, 1 \leq m \leq k$, also belong to $\mathscr{P}$. Since $\mathscr{D}$ has no oriented cycles, each $\tau_{A}$-orbit $\mathscr{O}$ of $\mathscr{D}$ contains a module which is the target of a path from $\mathscr{P}$. Moreover, since $\mathscr{D}$ is locally finite and admits infinitely many $\tau_{A}$-orbits, $\mathscr{P}$ contains paths of arbitrary large length. Then, from the above remarks, $\mathscr{P}$ contains a path $M=M_{0} \rightarrow M_{1} \rightarrow \cdots \rightarrow M_{n}$ of length $n$. Suppose that $\mathscr{C}$ contains a path $M_{i}=Z_{s} \rightarrow Z_{s-1} \rightarrow \cdots \rightarrow Z_{1} \rightarrow \tau_{A} M_{j}$ with $s \leq n, 0 \leq i, j \leq n$. Then, from our assumption on the length of walks from nonstable modules in $\mathscr{C}$ to $M$, we deduce that this path lies in $\mathscr{D}$. But this is impossible by the above property of paths from $\mathscr{P}$. Therefore, $\mathscr{C}$ does not contain paths of the form $M_{i}=Z_{s} \rightarrow Z_{s-1} \rightarrow \cdots \rightarrow Z_{1} \rightarrow \tau_{A} M_{j}$ with $s \leq n$, $0 \leq i, j \leq n$. Clearly, this implies that the path $M=M_{0} \rightarrow M_{1} \rightarrow \cdots \rightarrow M_{n}$ is sectional. Finally, we also infer that for any sectional path in $\mathscr{C}$ of the form $U_{t} \rightarrow U_{t-1} \rightarrow \cdots \rightarrow U_{1}=\tau_{A} M_{i+1} \rightarrow U_{0}=M_{i}$ with $1 \leq i \leq n-1, t \leq n$, the modules $U_{1}, \ldots, U_{t}$ are nonprojective and nonisomorphic to the modules $M_{0}, M_{1}, \ldots, M_{n}$. Then, by Lemma 3 , there is a cycle $M \rightarrow X_{1} \rightarrow \cdots \rightarrow X_{k} \rightarrow$ $M$ in $\bmod A$ with $X_{1}, \ldots, X_{k}$ from $\mathscr{C}$. This finishes the proof.

We shall also need the following lemma.

Lemma 5. Let $\mathscr{C}$ be a regular connected component of $\Gamma_{A}$ having only finitely many $\tau_{A}$-orbits. Assume that there is in $\bmod A$ a path

$$
X=Z_{0} \stackrel{f_{1}}{\rightarrow} Z_{1} \rightarrow \cdots \rightarrow Z_{m-1} \stackrel{f_{m}}{\rightarrow} Z_{m}=Y
$$

with $X$ and $Y$ from $\mathscr{C}$ and such that $f_{i}$ belongs to $\operatorname{rad}^{\infty}(\bmod A)$ for some $1 \leq i \leq m$. Then all modules in $\mathscr{C}$ are nondirecting.

Proof. Since $\mathscr{C}$ has only finitely many $\tau_{A}$-orbits, we infer by [4] that $\mathscr{C}$ consists of nonperiodic modules. Then, by [15], we have $\mathscr{C} \cong Z \Delta$, for some finite valued quiver $\Delta$ without oriented cycles. Let $M$ be a module in $\mathscr{C}$. We shall show that $M$ is nondirecting in $\bmod A$. Let $i$ be the least index such that $f_{i}$ belongs to $\operatorname{rad}^{\infty}(\bmod A)$. Then $Z_{0}, \ldots, Z_{i-1}$ belong to $\mathscr{C}$, and there is in $\mathscr{C}$ an infinite path

$$
Z_{i-1}=V_{0} \rightarrow V_{1} \rightarrow \cdots \rightarrow V_{s} \rightarrow V_{s+1} \rightarrow \cdots
$$


such that $\operatorname{rad}^{\infty}\left(V_{s}, Z_{i}\right) \neq 0$ for all $s \geq 0$. Since $\mathscr{C}$ has only finitely many $\tau_{A}$-orbits, there is a path in $\mathscr{C}$ of the form $M=U_{0} \rightarrow U_{1} \rightarrow \cdots \rightarrow U_{k}=V_{p}$ for some $p \geq 0$. Let $j$ now be the maximal index such that $f_{j}$ belongs to $\operatorname{rad}^{\infty}(\bmod A)$. Then $Z_{j}, \ldots, Z_{m}$ belong to $\mathscr{C}$, and there is in $\mathscr{C}$ an infinite path

$$
\cdots \rightarrow W_{r+1} \rightarrow W_{r} \rightarrow \cdots \rightarrow W_{1} \rightarrow W_{0}=Z_{j}
$$

such that either $\operatorname{rad}^{\infty}\left(Z_{j-1}, W_{r}\right) \neq 0$ for all $r \geq 0$, if $i<j$, or $\operatorname{rad}^{\infty}\left(V_{p}, W_{r}\right) \neq$ 0 for all $r \geq 0$, if $i=j$. Again, since $\mathscr{C}$ has only finitely many $\tau_{A}$-orbits, there is in $\mathscr{\mathscr { C }}$ a path of the form $W_{q}=L_{0} \rightarrow L_{1} \rightarrow \cdots \rightarrow L_{t}=M$ for some $q \geq 0$. Then we have in $\bmod A$ either a cycle

$$
\begin{aligned}
M & =U_{0} \rightarrow \cdots \rightarrow U_{k}=V_{p} \rightarrow Z_{i} \rightarrow \cdots \rightarrow Z_{j-1} \rightarrow W_{q} \\
& =L_{0} \rightarrow L_{1} \rightarrow \cdots \rightarrow L_{t}=M,
\end{aligned}
$$

if $i<j$, or a cycle

$$
M=U_{0} \rightarrow \cdots \rightarrow U_{k}=V_{p} \rightarrow W_{q}=L_{0} \rightarrow L_{1} \rightarrow \cdots \rightarrow L_{t}=M,
$$

if $i=j$. Therefore, $M$ is nondirecting in $\bmod A$.

\section{Proof OF THE THEOREM}

(i) Let $M$ be a directing module in the component $\mathscr{C}$. Then, by [4], $\mathscr{C}$ does not contain periodic modules, and therefore $\mathscr{C} \cong Z \Delta$ for some valued locally finite quiver $\Delta$ without oriented cycles, by [15] (and also [6]). Since $\mathscr{C}$ is regular and $M$ is a directing module in $\mathscr{C}$, we deduce from Lemma 4 that $\Delta$ is finite. Moreover, since $\mathscr{C}$ has no oriented cycles, from Lemma 5 we infer also that all modules from $\mathscr{C}$ are directing $A$-modules.

(ii) This is a direct consequence of (i) and Lemma 5.

(iii) From (i) we know that $\mathscr{C} \cong Z \Delta$ for some finite valued quiver $\Delta$ without oriented cycles. Let $U$ be the direct sum of modules corresponding to all vertices of a fixed $\Delta$ in $\mathscr{C}$. Then, by $[8,(1.2)]$, ann $U=\operatorname{ann} \mathscr{C}$ and clearly $U$ is a faithful module over $A / \operatorname{ann} \mathscr{C}$. On the other hand, $t_{Q}(A)$ is contained in ann $\mathscr{C}$ and $U$ is a sincere $B$-module, where $B=A / t_{Q}(A)$. We claim that $U$ is not the middle term of a short chain in $\bmod B$; that is, for any indecomposable $B$-module $Z$, either $\operatorname{Hom}_{B}(Z, U)=0$ or $\operatorname{Hom}_{B}\left(U, \tau_{B} Z\right)=0$. Suppose that $\operatorname{Hom}_{B}(Z, U) \neq 0$ and $\operatorname{Hom}_{B}\left(U, \tau_{B} Z\right) \neq 0$ for some indecomposable $B$ module $Z$. Then there are indecomposable direct summands $X$ and $Y$ of $U$ and a path in $\bmod A$ of the form $X \stackrel{f}{\rightarrow} \tau_{B} Z \rightarrow W \rightarrow Z \stackrel{g}{\rightarrow} Y$. Observe that if $Z$ belongs to $\mathscr{C}$ then $\tau_{B} Z=\tau_{A} Z$, because $\mathscr{C}$ consists entirely of $B$-modules. Now, since $\mathscr{C} \cong Z \Delta, \Delta$ has no oriented cycles, and $X, Y$ lie on some fixed $\Delta$, we infer that one of the maps $f$ or $g$ belongs to $\operatorname{rad}^{\infty}(\bmod A)$. But then, by Lemma 5, all modules from $\mathscr{C}$ are nondirecting, a contradiction. Consequently, $U$ is not the middle term of a short chain in $\bmod B$ and then, by $[7,(3.1)]$, $U$ is a faithful $B$-module. This proves that $t_{Q}(A)=\operatorname{ann} U=\operatorname{ann} \mathscr{C}$.

(iv) and (v). We know that $U$ is a faithful $B$-module. Moreover, we infer, by Lemma 5, that $\operatorname{Hom}_{B}\left(U, \tau_{B} U\right)=0$ and $\operatorname{Hom}_{B}\left(\tau_{B}^{-} U, U\right)=0$. Then applying $[8,(1.5)]$ and its dual, we obtain that $p d_{B} U \leq 1$ and $i d_{B} U \leq 1$. Clearly, $\operatorname{Ext}_{B}^{1}(U, U) \cong \operatorname{DHom}_{B}\left(U, \tau_{B} U\right)=0$. Finally, if $\operatorname{Hom}_{B}(U, X) \neq 0$ for some indecomposable $B$-module $X$ which is not a direct summand of $U$, then $\operatorname{Hom}_{B}\left(\tau_{B}^{-} U, X\right) \neq 0$. Consequently, by $[8,(1.6)], U$ is a tilting and 
cotilting $B$-module. Then, by (ii), $H=\operatorname{End}_{B}(U)$ is a hereditary algebra of type $\Delta^{\text {op }}$. Hence $B=\operatorname{End}_{H}(T)$ for a tilting $H$-module $T$ and $\mathscr{C}$ is a connecting component of $\Gamma_{B}$. Since $\mathscr{C}$ is regular, then, by [10, p. 42;11, Theorem], $T$ is a regular tilting $H$-module. Moreover, in this case $B$ is not a concealed algebra (see [9]) and $\mathscr{C}$ is the unique connecting component of $\Gamma_{B}$.

\section{Proofs of Corollaries 1,2 , and 3}

Proof of Corollary 1. The claim is obvious in the case where $\mathscr{C}$ is a stable tube. If $\mathscr{C}$ is not a stable tube, then by $[4,15], \mathscr{C}$ has no oriented cycles and our claim is a direct consequence of Lemma 4.

Proof of Corollary 2. Observe first that $A$ admits only finitely many ideals of the form $t_{Q}(A)$, where $Q$ is a direct summand of $A$. Hence, by the theorem, $\Gamma_{A}$ admits at most finitely many connected components containing directing modules. Hence, it is enough to show that any connected component of $\Gamma_{A}$ admits at most finitely many $\tau_{A}$-orbits containing directing modules. Suppose that $\mathscr{C}$ is a connected component of $\Gamma_{A}$ which admits infinitely many $\tau_{A^{-}}$ orbits containing directing modules. Then, since $\mathscr{C}$ is locally finite, there is a connected component $\mathscr{D}$ of the stable part $\mathscr{C}_{s}$ of $\mathscr{C}$ which admits infinitely many $\tau_{A}$-orbits containing directing modules. Moreover, since the number of nonstable $\tau_{A}$-orbits in $\mathscr{C}$ is finite, there exists a directing module $M$ in $\mathscr{D}$ such that the length of any walk in $\mathscr{C}$ from a nonstable module to $M$ is at least $2 n$. But then, from Lemma $4, M$ is nondirecting, a contradiction. Therefore, each connected component of $\Gamma_{A}$ admits at most finitely many $\tau_{A^{-}}$ orbits containing directing modules, and hence $\Gamma_{A}$ has the same property. This finishes our proof.

Proof of Corollary 3. It was proved in [5] that a finite-dimensional algebra $A$ over an algebraically closed field $k$ is representation-finite if and only if $\Gamma_{A}$ has only finitely many $\tau_{A}$-orbits. Then Corollary 3 is a direct consequence of Corollary 2.

The author was recently informed by Idun Reiten that, independently, L. Peng and J. Xiao have also proved Corollary 2.

\section{ACKNOWLEDGMENT}

These results were obtained while I was visiting the University of Tsukuba. I would like to thank $\mathrm{H}$. Tachikawa for the invitation and all colleagues in Tsukuba for their hospitality during my stay. I would also like to thank the referee for remarks concerning the presentation of the results included in the paper.

\section{REFERENCES}

1. M. Auslander and I. Reiten, Representation theory of artin algebras. III; IV, Comm. Algebra 3 (1975), 239-294; 5 (1977), 443-518.

2. R. Bautista and S. O. Smalø, Nonexistent cycles, Comm. Algebra 11 (1983), 1755-1767.

3. K. Bongartz, Tilted algebras, Representations of Algebras, Lecture Notes in Math., vol. 903, Springer, Berlin, 1981, pp. 26-38.

4. D. Happel, U. Preiser, and C. M. Ringel, Vinberg's characterization of Dynkin diagrams using subadditive functions with applications to DTr-periodic modules, Representation Theory. II, Lecture Notes in Math., vol. 832, Springer, Berlin, 1980, pp. 280-294. 
5. S. Liu, The degrees of irreducible maps and shapes of Auslander-Reiten quivers, J. London Math. Soc. (2) 45 (1992), 32-54.

6. __ Semi-stable components of an Auslander-Reiten quiver, Preprint No. 12/91, University of Trondheim.

7. I. Reiten, S. O. Smalø, and A. Skowroński, Short chains and short cycles of indecomposable modules, Proc. Amer. Math. Soc. 117 (1993), 343-354.

8. __ Short chains and regular components, Proc. Amer. Math. Soc. 117 (1993), 601-612.

9. C. M. Ringel, Tame algebras and integral quadratic forms, Lecture Notes in Math., vol. 1099, Springer, Berlin, 1984.

10. _ Representation theory of finite-dimensional algebras, Representations of Algebras (Proceedings, Durham 1985) Cambridge Univ. Press, London and New York, 1986, pp. 7-80.

11. _ The regular components of the Auslander-Reiten quiver of a tilted algebra, Chinese Ann. Math. Ser. B 1 (1988), 1-18.

12. A. Skowroński, Cycles of indecomposable modules, Representation Theory of Finite Groups and Finite Dimensional Algebras (Proc. Res. Inst. Math. Sci., Kyoto 1991) J. Math. Soc. Japan (to appear).

13. __ Generalized standard Auslander-Reiten components, J. Math. Soc. Japan (to appear).

14. A. Skowroński and S. O Smalø, Directing modules, J. Algebra 147 (1992), 137-146.

15. Y. Zhang, The structure of regular components, Canad. J. Math. 43 (1991), 652-672.

Institute of Mathematics, Nicholas Copernicus University, Chopina 12/18, 87-100 TORUŃ, POLAND

E-mail address: skowron@mat.torun.edu.pl 